\title{
Microphysics in Astrophysical Plasmas
}

\author{
Steven J. Schwartz • Ellen G. Zweibel • Martin Goldman
}

Received: 25 January 2013 / Accepted: 8 March 2013 / Published online: 18 May 2013

(c) The Author(s) 2013. This article is published with open access at Springerlink.com

\begin{abstract}
Although macroscale features dominate astrophysical images and energetics, the physics is controlled through microscale transport processes (conduction, diffusion) that mediate the flow of mass, momentum, energy, and charge. These microphysical processes manifest themselves in key (all) boundary layers and also operate within the body of the plasma. Crucially, most plasmas of interest are rarefied to the extent that classical particle collision length- and time-scales are long. Collective plasma kinetic phenomena then serve to scatter or otherwise modify the particle distribution functions and in so-doing govern the transport at the microscale level. Thus collisionless plasmas are capable of supporting thin shocks, current sheets which may be prone to magnetic reconnection, and the dissipation of turbulence cascades at kinetic scales. This paper lays the foundation for the accompanying collection that explores the current state of knowledge in this subject. The richness of plasma kinetic phenomena brings with it a rich diversity of microphysics that does not always, if ever, simply mimic classical collision-dominated transport. This can couple the macro- and microscale physics in profound ways, and in ways which thus depend on the astrophysical context.
\end{abstract}

Keywords Microphysics · Plasmas · Astrophysics · Space plasmas

S.J. Schwartz ( $\square)$

Blackett Laboratory, Imperial College London, South Kensington, London SW7 2AZ, UK

e-mail: s.schwartz@imperial.ac.uk

E.G. Zweibel

Astronomy Department, University of Wisconsin, Madison, WI 53706, USA

e-mail: zweibel@astro.wisc.edu

M. Goldman

Department of Physics, University of Colorado, Boulder, CO 80309-0390, USA

e-mail: Martin.Goldman@Colorado.EDU 


\section{Introduction}

The astrophysical world is filled with plasmas, from the solar atmosphere through supernova remnants to distant galaxies. Despite these diverse environments, there are common underlying physical mechanisms at work. Shock waves form at flow interaction regions, current layers breakdown to release bottled-up magnetic energy, and turbulence tangles magnetic fields and cascades energy to small scales where it is dissipated.

In the classical view, these and many more phenomena are controlled by transport processes (diffusion, conduction, etc.) that are mediated by inter-particle collisions. The resulting collision frequencies and transport coefficients are then used in a fluid approach to the physics. In such an approach, these coefficients depend only on the local state parameters (e.g., density, temperature) independent of the large-scale region of interest. In this view, the media never stray far from Maxwellian thermodynamic equilibrium. If we lived in a fluid Universe, there would be no solar flares, no ultra-relativistic cosmic rays, and no Aurora Borealis.

However, most astrophysical plasmas are too rarefied for binary Coulomb collisions to be effective. That is, the characteristic length- and time-scales are too short for the infrequent collisions to maintain thermodynamic equilibrium and control the transport processes. Instead, the collective interaction of the plasma particles and fields results in a range of phenomena that dominate the microphysics. The absence of collisions opens up the possibility for some particles to be accelerated to become the highest energy cosmic rays while their neighbours participate in a less spectacular background plasma.

Thus the collective interactions operate selectively on the plasma particles, depending on their species, their location in velocity space (e.g., through resonant wave-particle interactions), or the pre-existence of fluctuations in electromagnetic fields. None of these are necessarily prescribed by the gross macroscopic conditions such as density or temperature or their gradients, and so the resulting description of the transport processes probably bears little resemblance to the classical collision-dominated one.

Early attempts to attribute the action of wave-particle interactions as some "anomalous resistivity $\eta$," for example Papadopoulos (1977), in which

$$
\mathbf{j}=\mathbf{E} / \eta
$$

while convenient are probably rarely accurate. In the first instance, even if this linear relationship holds, $\eta$ could be a function of details in the particle distribution functions $f_{i}(\mathbf{v})$ and not simply density and temperature. And secondly, the nonlinear relationship between current and electric field could involve the global context including the total current, the contribution of particles traversing macroscopic scales to the local population, or DC electric or magnetic fields unrelated to the current which nonetheless influence the collective behaviour.

Because there is no single, general description, these non-classical forms are best illustrated through specific examples. In this paper, we will describe the typical applications to shock waves, magnetic reconnection, and turbulence. Those applications will be developed in the accompanying papers in this volume. We will see that some aspects of the microphysics are very specific to the parameter regimes involved while others are more universal, at least qualitatively. Accordingly, the juxtaposition of laboratory, solar system, and astrophysical applications should lead to important cross-fertilisation. We will also see that the microphysics couples into the macrophysics in profound ways that have no counterparts in classical transport theory. 


\section{Common Themes}

Before discussing specific applications, we lay out a few common themes. These illustrate the origins of the departure from classical transport and some key features to consider in any context.

\subsection{Plasma Hierarchy}

It is instructive to re-examine the basis of transport and dissipation in a plasma. We begin with the Boltzmann equation for the one-particle distribution function $f_{i}(\mathbf{x}, \mathbf{v}, t)$ for a species $i$ :

$$
\frac{\partial f_{i}}{\partial t}+\mathbf{v} \cdot \frac{\partial f_{i}}{\partial \mathbf{x}}+\mathbf{a} \cdot \frac{\partial f_{i}}{\partial \mathbf{v}}=\left(\frac{\partial f_{i}}{\partial t}\right)_{C}
$$

where the right hand side is a placeholder for collisions and other processes which are not represented within the particle acceleration a and which may alter the phase space density in an instantaneous or discontinuous manner not representable in this differential formulation. Such processes include interactions with other species, ionisation, etc.

Taking velocity-space moments of (1) results in an infinite hierarchy of equations for the density, velocity, pressure tensor, heat flux, ..., associated with the species. For example, multiplying by $m_{i} \mathbf{v}$ and integrating over velocities yields the momentum equation:

$$
\frac{\partial\left(\rho_{i} \mathbf{V}_{i}\right)}{\partial t}+\nabla \cdot \underline{\underline{P}}_{i}-\mathbf{F}_{i}=\left\langle m \mathbf{v}\left(\frac{\partial f_{i}}{\partial t}\right)_{C}\right\rangle
$$

One obvious problem is that this requires knowledge of the next higher moment (the stress tensor $\underline{\underline{P}}_{i}$ ) so that ultimately some closure assumption is required. The microphysical processes $\overline{r e s p o n s i b l e ~ f o r ~ m o m e n t u m ~ t r a n s p o r t ~ m a n i f e s t ~ t h e m s e l v e s ~ i n ~ t w o ~ t e r m s ~ o f ~(2) . ~ F i r s t l y, ~}$ the right hand side contains all the interactions with other species, collisions, etc. This gives rise to the normal collisional coupling between species, and the viscosity, when particular forms of the collision operator are employed, as in the case of near-equilibrium collisional plasmas (Braginskii 1965).

Additionally, the force density $\mathbf{F}_{i}$ on the species as a whole can be thought of as arising from two contributions. One is derived from the acceleration a in (1) due to macroscopic, quasi-steady fields and particle distributions. The second is the correlation of any fluctuating acceleration $\delta \mathbf{a}$ with fluctuations $\delta f_{i}$. This nonlinear convolution has a non-zero average. In instability analyses this captures the nonlinear wave-particle interaction contributions to the momentum transport. Similar correlations appear when the stress-tensor $\underline{\underline{P}}_{i}$ is recast in terms of the mean velocity (Che et al. 2011). There is no reason, of course, for these nonlinear contributions, even when spatially averaged or smoothed, to resemble the viscous or collisional species coupling terms. Wave-particle interactions act differently upon, e.g., resonant and non-resonant particles, shaping $f_{i}$ in ways that cannot be determined by the macroscopic fluid parameters but which nonetheless contribute to the transport of, in this example, the momentum.

Another fundamental aspect of the microphysics is revealed in the $m v^{2} / 2$ moment of (1) which yields an energy equation. In addition to dissipative processes which heat the species and clearly depend on the microphysics, the hierarchy of moment equations is often truncated at this level by some closure assumption, e.g., that the heat flux is zero, which turns this moment equation into an equation of state of the form

$$
\left(\frac{\partial}{\partial t}+\mathbf{V} \cdot \frac{\partial}{\partial \mathbf{x}}\right)\left(P_{i} \rho_{i}^{-\gamma}\right)=\text { dissipative term }
$$


where the term on the right hand side can be identified with the right hand side of (1) together with any nonlinear response to fluctuations in the plasma and fields. Now much of the unknown microphysics is collapsed into the unknown, and presumed constant, adiabatic in$\operatorname{dex} \gamma$ reflecting the equation of state for species $i$ or, in the case of single fluid descriptions, for the plasma as a whole. This is a convenient but rarely appropriate prescription for the influence of the microphysics in transporting energy. Similar concerns arise in the derivation of the double-adiabatic equations for an anisotropic plasma (Chew et al. 1956), which rely on macroscopic conditions related to the first and second adiabatic invariants of particles in a magnetic field; conditions which are rarely realised in practise.

\subsection{Coupling of Scales}

All dynamical media involve disparate scales which couple to one another (Schwartz et al. 2009a, 2009b). In viscous fluids, the thin boundary layers are driven by, and strongly influence, the macroscopic flow field. Collisionless plasmas contain many more scales, ranging from the macroscopic context through energetic particle scales to thermal ion and electron scales. Additionally, there are magnetic scales linked to particle gyromotion and electric scales linked to charge separation, collisionless inertial lengths or skin depths (e.g., $c / \omega_{p i, e}$ where $\omega_{p i, e}$ is the plasma frequency), and Debye lengths. Fluctuations and turbulence can lead to broad probability distribution functions of pitch angle scattering times and lengths. These imply a nonlocal random walk, which can lead to nondiffusive transport.

Small-scale breakdown of thin current layers in the solar atmosphere, for example, can lead to the eruption of solar flares and the reconfiguration of the magnetic field topology over vast distances. Electromagnetic fields confined to thin layers at a collisionless shock can inject energetic particles into the unshocked medium where locally-driven turbulence can scatter and further accelerate those particles, leading to a feedback process by which large-scale shocks are mediated by self-generated cosmic rays that account for a significant fraction of the shock energetics.

\subsection{Micro-instabilities}

The non-equilibrium aspects of collisionless plasmas opens up the possibility for differential flows (currents or beams), temperature anisotropies in which the kinetic temperature along and perpendicular to the magnetic field, say, are different, and other kinetic features (e.g., "bumps on tail", loss cones, ring distributions). If the removal of such features would lead to a lower energy state, they represent sources of "free energy" capable of driving plasma micro-instabilities (Gary 2005). This is another example of scale coupling, since the free energy is usually driven by macroscopic interactions or sources of some kind.

In a micro-unstable plasma, particles in resonant regions of velocity-space coherently interact with electrostatic or electromagnetic perturbations which grow at the expense of the resonant particles. In the nonlinear stages one expectation is that the unstable features (beam, anisotropy or whatever) will diminish toward marginal instability, with the free energy released residing in the fluctuating fields and non-resonant populations. Eventually that fluctuation energy would damp heating the plasma. Alternatively, nonlinear wave cascades can remove fluctuation energy before it can modify the free-energy regions of phase space; such a competition, as well as particle trapping, can yield a steady state that is not welldescribed as being marginally stable against the original free energy source.

Two heuristic scenarios are commonly invoked. In "anomalous transport" theory, the nonlinear interactions are cast into the form of a collision term with the collision frequency 
Fig. 1 Occurrence frequency (color scale) of solar wind plasma in proton temperature anisotropy $\left(T_{\perp} / T_{\|}\right)$vs. parallel plasma beta $\left(\beta_{\|}\right)$from Matteini et al. (2007). The curves show thresholds for small, positive growth rates near marginal stability for the ion-cyclotron/mirror mode (upper curves) and firehose instability (lower curves). The steeply falling dash-dot-dot-dot curve in (a) is the prediction based on conservation of particle magnetic moments. This figure suggests that these instabilities constrain the thermal properties of the expanding solar wind, which would otherwise be driven, e.g., to small anisotropy values through the action of adiabatic particle invariants

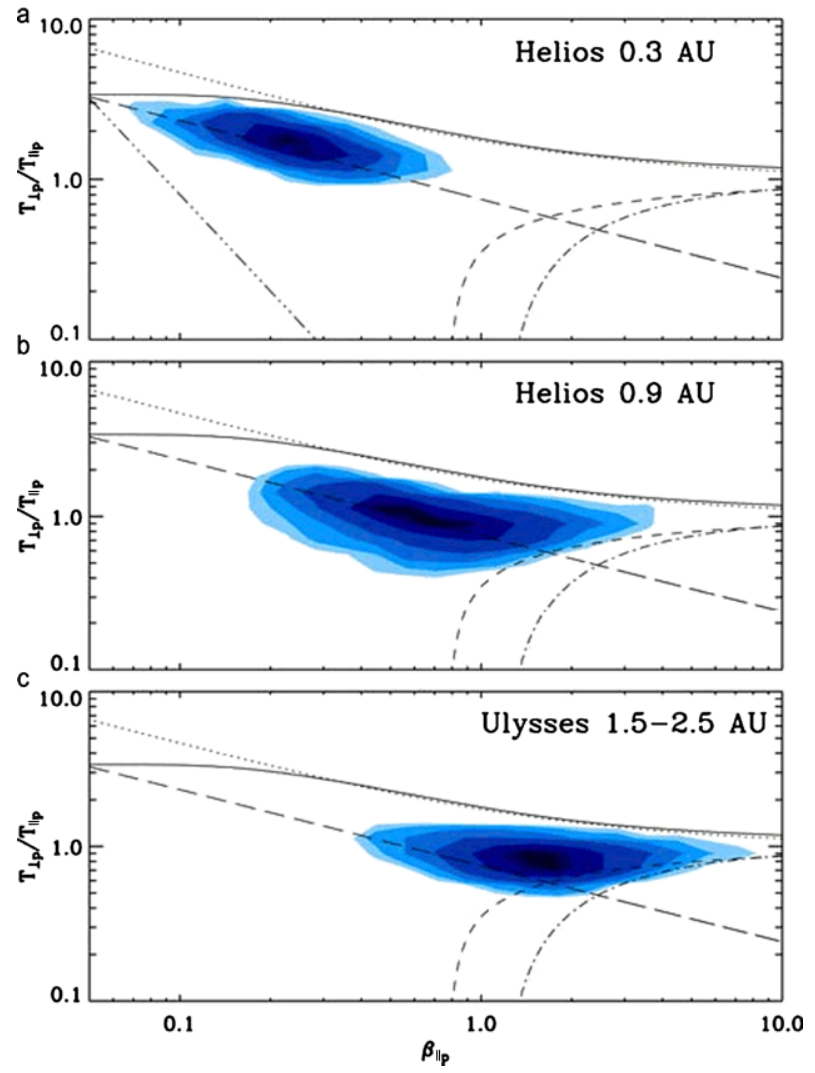

dependent on the intensity of the fluctuations. Thus, for example, the influence of an instability driven by an electric current is made to look in form like that due to classical resistivity, with the classical collision frequency replaced by the nonlinear wave-particle scattering rate. Similarly, the transport of streaming cosmic rays is influenced by effective wave-particle scattering arising from self-generated Alfvén waves due to the cosmic ray streaming instability (Skilling 1975), or by scattering due to pre-existing levels of turbulence.

Since kinetic instabilities grow on short, kinetic timescales, another approach when considering the larger scale consequences of micro-instabilities is to assume that the plasma can never be grossly unstable. In this case the plasma parameters (beam speed, anisotropy, or other free energy source) should be close to, or below, values corresponding to marginal stability. Figure 1 shows that the solar wind temperature anisotropy and plasma beta appear to be constrained within the near-marginally stable limits of the mirror and firehose instabilities (Matteini et al. 2007).

Such constraints can be employed directly in macroscopic models rather than attempts to predict the instability nonlinear wave intensities and corresponding effective collision frequencies. This prescription can only assume that when the macroscopic conditions bring the plasma into the stable regime the microphysical processes cease to operate. While both approaches to incorporate the action of micro-instabilities into a macroscopic description of the plasma are useful, both fall far short of a self-consistent approach to the micro-macroscale problem which remains largely unsolved. 
Fig. 2 The formation of a DC electric field (and current) in the boundary between a flowing plasma and a vacuum magnetic field (after Cowley 1995 Fig. 1)

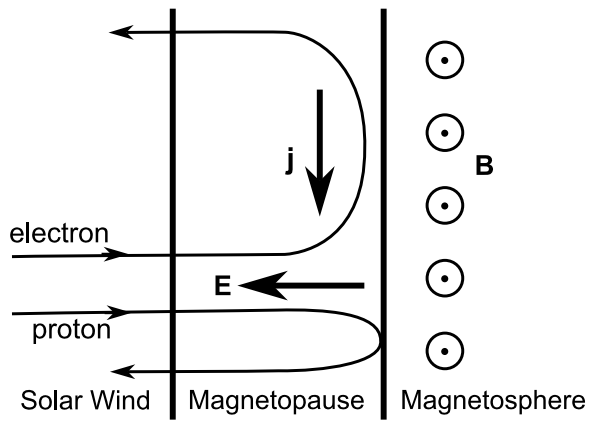

\section{Shocks and Discontinuities}

Despite the collisionless nature of most astrophysical plasmas, the collective self-consistent behaviour of the particles together with the influence of large-scale magnetic fields in inhibiting cross-field motion prevents wholesale intermingling of plasmas from different sources. Instead, the "Plasma Universe" (Alfvén 1986) is divided into cells of plasma separated by relatively sharp boundaries or discontinuities. Supersonic flows also drive nonlinear macroscopic shock waves, examples of which range from the relatively weak shocks driven by high speed streams or planetary obstacles in the solar wind flow (Schwartz 2006) through shocks driven by supernova explosions in the interstellar medium to shocks in galaxy clusters driven by extragalactic jets or large scale accretion flows.

These discontinuities are maintained through the role of microphysics in supporting the currents, DC electromagnetic fields, and transport processes within them. Here we provide a few illustrations of the microphysical processes found at such discontinuities.

\subsection{Fields}

It can be easy to overlook the DC electric fields at discontinuities and to underestimate the subtle microphysical processes and balances required to support them. Consider, for example, an unmagnetised plasma impinging transversely on a vacuum magnetic field as first studied by Chapman and Ferraro (1931) (see Cowley 1995 for a review) in the context of the Earth's magnetopause. While both ions and electrons will be turned around by the magnetic field, the ions will attempt to penetrate further owing to their larger momentum and hence gyroradius. That would lead to a large charge separation over the turnaround layer. Since the ion gyroradius is typically much larger than a Debye length, this charge separation must be inhibited through the establishment of an electric field which opposes the ion motion, enhances the electron penetration (and local gyroradii), and results in a layer of thickness $c / \omega_{p e}$ carrying an appreciable electric current as sketched in Fig. 2.

Similarly, collisionless shocks need to dissipate the incident kinetic energy through some microphysical process. Treumann (2009) provides a very comprehensive review of shock physics. Above a critical Mach number, which is only 2.8 or less depending on the shock parameters, the ions and electrons are heated by different mechanisms both of which rely on the DC fields within the shock layer. An electrostatic field is maintained by the electron temperature gradient. This, in concert with the magnetic field profile, reflects a fraction of the incident ions which gyrate back into the unshocked medium, but are convected back into and ultimately downstream. There they form a partial ring gyrating about the directly transmitted ions as sketched in Fig. 3 (see, e.g., Burgess 1995). This distribution of particles, 
Fig. 3 Positional space (top) and velocity space (bottom) signatures of ions reflected at a shock surface (after Sckopke et al. 1983). The combination of directly transmitted and initially reflected particles downstream of the shock location results in a distribution that has slower net bulk flow speed and larger velocity spread (and hence kinetic temperature)

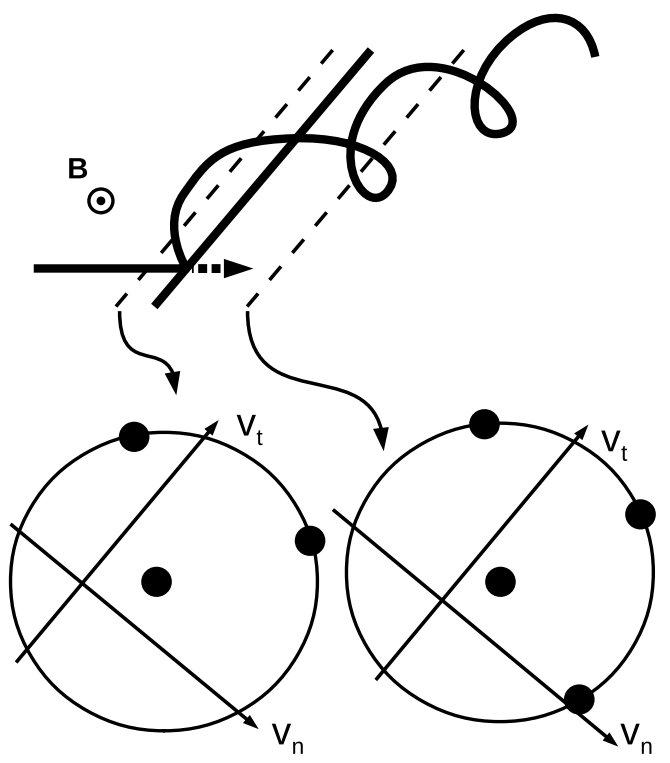

though far from equilibrium, has a lower bulk velocity and higher velocity spread than the incident plasma; that is, it has been slowed and "heated" entirely by the action of the DC fields without any scattering or collisions.

At the same time, this electric field causes the electron population to bifurcate into an incident population, which gains energy, and an escaping heated population, which loses energy during that escape and therefore originates downstream from a higher energy region in velocity space (Scudder et al. 1986). Thus the action of the DC field on the electrons results in a wide distribution downstream with a 'hole' at low energies. The consequence is that the DC fields are able to account for the inflation in velocity space expected through a heating mechanism.

Recently, Mitchell et al. (2012) investigated the added complication of electron heating at curved shocks, such as the bow shock formed by the impingement of the solar wind on the Earth's magnetosphere. Under collisionless conditions, electrons encountering the shock DC fields at one location traverse the region of shocked plasma to re-encounter the shock at a different location. This "cross-talk" connects portions of the curved shock at which the local conditions (e.g., Mach number, shock geometry) and hence total heating requirements are very different. Mitchell et al. found that the collisionless transport results in the entire shocked electron population being nearly isothermal, implying that the ion heating must vary greatly in order to balance the total energy budget around the shock surface. This work reveals a complex interplay amongst the DC fields (supported by the near-isothermal shocked electrons), local shock conditions, and global shock shape. In particular, for such shocks knowing the local shock parameters is insufficient to predict the energy partition amongst plasma species. Once again, there is strong coupling between different scales in the plasma.

\subsection{Instabilities}

In the shock example above, both the ion and electron distributions, as described, contain considerable free energy capable of driving micro-instabilities which would be ex- 
pected to fill the electron 'hole' (together with electrons trapped downstream by the cross-shock electrostatic potential) and mix the transmitted/reflected ion components. Thus micro-instabilities finish the thermalisation process. See, e.g., Schwartz et al. (1996) and McKean et al. (1992) for discussion of ion waves and instabilities, of which the Alfvén ion cyclotron and mirror modes are the most commonly invoked. Electrons interact with preexisting waves driven by the ion kinetics (e.g., the lower hybrid drift instability) or drive whistler or other electron-scale waves directly (e.g., Wu et al. 1984; Tsurutani et al. 1982; Masood et al. 2006).

There are many more examples of micro-instabilities associated with shocks and discontinuity layers. Some of these participate in acceleration processes.

\subsection{Acceleration}

While microinstabilities can thermalize the core of the particle distribution function, they can also extract a high energy tail. Galactic cosmic rays, which represent $10^{-9}-10^{-10}$ of interstellar particles but carry about as much energy as the thermal gas, dramatically exemplify this. Although the association between supernovae and cosmic rays had long been suspected, a viable mechanism was not suggested until the 1970's, when Bell (1978) and Blandford and Ostriker (1978) independently developed a theory for first order Fermi acceleration of cosmic rays by strong interstellar shocks driven by supernovae. In the original theory, the shock was assumed to be quasi-parallel, and was idealized as a discontinuity with the upstream and downstream flow properties connected by the Rankine-Hugoniot relations. In the shock frame, unshocked fluid streams into the shock at speed $V_{s}$ and streams out at speed $V_{s} / R$, where $R$ is the compression ratio of the shock. A particle with momentum $p$ traveling upstream from the shock gains energy $2 p V_{s}$ if scattered back downstream, and loses energy $2 p V_{s} / R$ if scattered back upstream, resulting in a net energy gain of $2 p V_{s}(1-1 / R)$ per loop. The resulting spectrum in momentum space is a power law; $p^{-3 R /(R-1)}$. For strong non-relativistic shocks, $R \sim 4$ leading to a power law $\propto p^{-4}$. Propagation through the interstellar medium steepens this spectrum, bringing it closer to the observed $p^{-4.7}$ spectrum.

Electromagnetic fluctuations generated by microinstabilities play two roles in this scenario: scattering particles back and forth across the shock, and keeping the distribution of particle pitch angles nearly isotropic. The fluctuations are thought to be Alfvén waves which interact with the cosmic rays through gyroresonance, and their primary source is thought to be an instability driven by super-Alfvénic streaming of the cosmic rays themselves (Wentzel 1968; Kulsrud and Pearce 1969). Later it was realized that including the momentum and energy of cosmic rays, the stresses on the fluid exerted by the waves (Dewar 1970), and the energy deposited in the fluid by wave dissipation modifies the Rankine-Hugoniot relations and broadens the shock by creating a cosmic ray precursor (Voelk et al. 1984). When these effects, and the increase in scattering mean free path with cosmic ray energy (which causes higher energy particles to "see" a larger velocity jump and gain more energy per loop), are accounted for, the power law expected from the simple theory is replaced by a more complicated distribution (Blasi 2012; Reville et al. 2009).

This Fermi acceleration process has been observed, though at non-relativistic energies, in situ e.g., at the Earth's bow shock. Figure 4 shows an experimentally determined upstream e-folding distance (related to the wave-scattering mean free path) as a function of particle energy as deduced by Kis et al. (2004). A long-standing feature of this theory is the requirement to "inject" suprathermal particles into this accelerator. It would appear, at least for low Mach number shocks, that processes within the shock layer are able to select, energise, and 
inject particles directly from the incident thermal population, as shown in Fig. 5. In astrophysical environs, any pre-existing suprathermal population would similarly inject particles into the Fermi process. Injected particles must be able to travel upstream against the incident flow to participate in the acceleration mechanism.

The gyroresonant streaming instability dominates when the cosmic ray energy density $U_{c r}$, background magnetic field energy density $U_{B}$, and cosmic ray drift speed $v_{D}$ satisfy the inequality $U_{c r} / U_{B}<c / v_{D}$. When the inequality is reversed, a nonresonant instability driven by the thermal electron return current grows faster and drives electromagnetic fluctuations which differ significantly from Alfvén waves (Zweibel 2003; Bell 2004). PIC simulations of the instability suggest that it amplifies the magnetic field significantly, increasing the rate of

Fig. 4 Observationally determined exponential scale for Fermi-accelerated ions at the Earth's bow shock, from Kis et al. (2004)
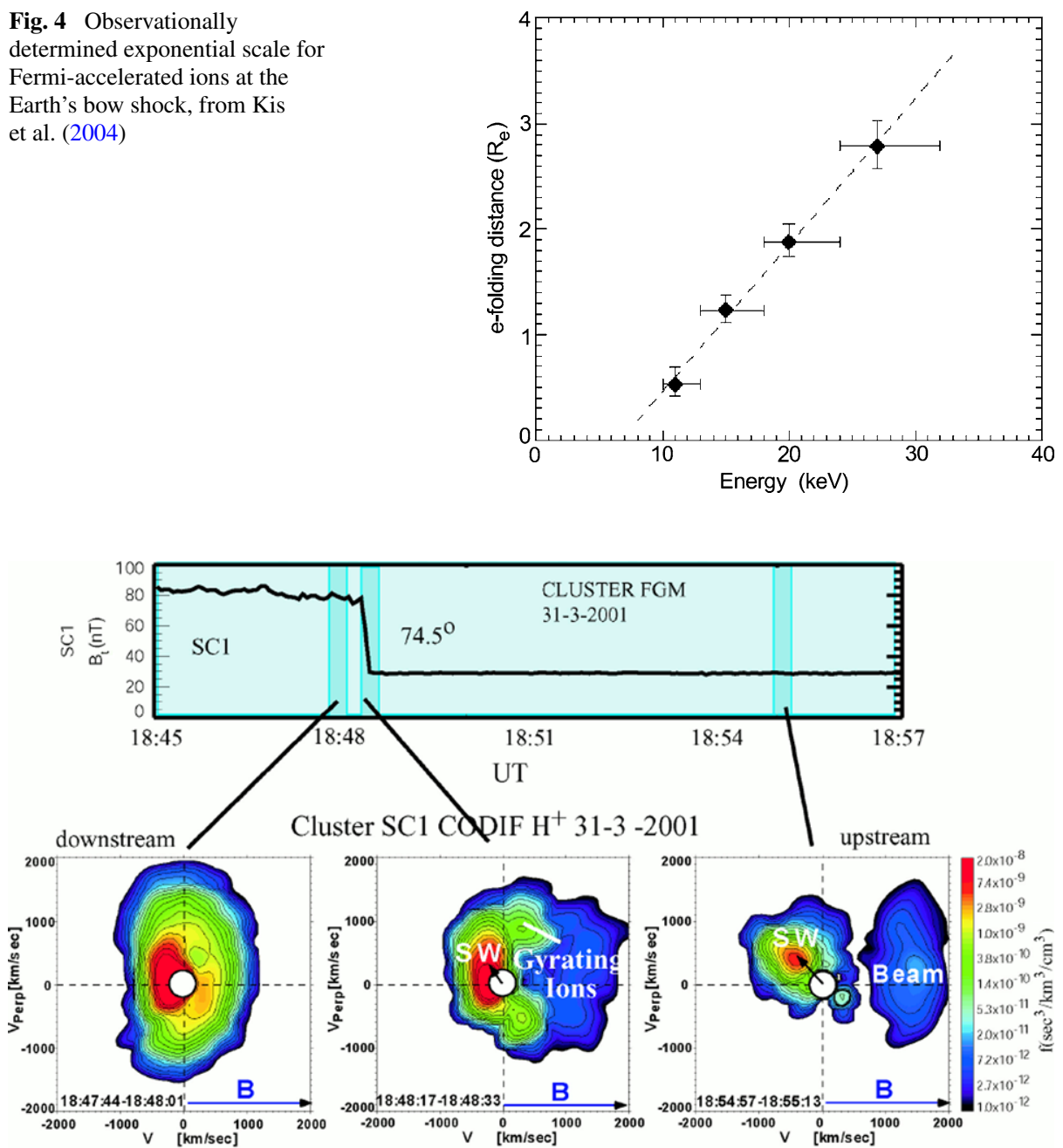

Fig. 5 Formation of ion beams via coherent process at the Earth's bow shock, from Kucharek et al. (2004). Note that the beam occupies regions of velocity space that are empty in the downstream region, thus establishing that the beam must originate at the shock itself. This is at least a partial answer to the "injection problem" for cosmic rays 
shock acceleration and the maximum energy to which it can accelerate particles (Riquelme and Spitkovsky 2010; Ohira et al. 2009).

The cosmic ray acceleration picture cannot be tested with in situ probes the way solar system acceleration can, but there are predictions that can be checked remotely. Among them are the cosmic ray spectrum itself, the modification of the Rankine-Hugoniot relations (especially the postshock temperatures), the magnetic field strengths, and the cosmic ray energy densities and maximum energies in the shock itself.

Electron acceleration (as opposed to heating) is harder due to lack of efficient waveparticle scattering and more rapid energy losses. These effects must come into play in Galactic cosmic ray acceleration; electrons comprise only 1-2\% of primary cosmic rays. It is important to be able to estimate the relative efficiencies of cosmic ray electron and ion acceleration, as the electron component is far easier to detect remotely through its synchrotron and inverse Compton emission. There is evidence for coherent reflection at shocks which can lead to significant one-step energisation (Wu 1984; Leroy and Mangeney 1984). These processes exploit the fact that the electron thermal distribution is much wider in velocity due to the electron mass. As a result, simple magnetic mirroring in the frame in which the shock is at rest and the incident flow is field-aligned (removing the $-\mathbf{V} \times \mathbf{B}$ electric field), the deHoffmann-Teller frame, yields an energetic beam with appreciable density. There are also recent reports of Fermi-accelerated relativistic electrons at the Saturn's bow shock (Masters et al. 2013). Within the solar system, the Fermi process is limited by the finite size of planetary bow shocks which results in particle escape. Thus the relatively large size of the Saturnian bow shock is more favorable for acceleration to higher energies given appropriate solar wind conditions.

\section{Collisionless Magnetic Reconnection}

Magnetic reconnection is a ubiquitous energetic plasma process commonly found in planetary magnetospheres, in the solar corona, in the solar wind, in astrophysical objects (Zweibel and Yamada 2009) and in laboratory plasmas (for a review, see Yamada et al. 2010). Reconnection is of special interest because it can convert large amounts of magnetic energy stored on both sides of a current sheet into particle energy. The energetic particles, instabilities and/or radiation associated with reconnection can disrupt confinement of fusion plasmas in toroidal devices (Yamada et al. 1994) and can initiate energetic particle flows from the Sun to Earth (Forbes and Priest 1995). Dungey (1961) introduced the idea that reconnection at the Earth's magnetopause (and within the geomagnetic tail) could drive the aurora. We now know that the energised particles populate large regions of the Earth's magnetosphere (Dungey 1995; Vasyliunas 1975; Kivelson and Russell 1995) where they may affect communications and possibly pose a threat to spacecraft or astronauts.

\subsection{How Does Topological Reconnection Begin?}

One major problem in the physics of magnetic reconnection is to understand how oppositelydirected components of magnetic field lines break and reconnect. There are various ways in which this can happen. In dense plasmas reconnection can be enabled by particle collisions. An example is reconnection near the Sun's photosphere. Collisional reconnection can be modeled by resistive MHD (but not ideal MHD). However in Earth's magnetopause and magnetotail, and in many planetary and astrophysical environments, the plasma is collisionless, entropy is conserved, and modeling reconnection often requires kinetic simulations, such as Particle In Cell (PIC) simulations. 
Collisionless reconnection in Earth's magnetopause is asymmetric-with different ambient conditions on the two sides of the current sheet. In Earth's magnetotail it is generally symmetric in the two lobes on either side of the plasmasheet. A simple initial condition often employed in kinetic simulations of symmetric reconnection consists of a uniform thin current sheet separating oppositely-directed straight magnetic field lines which asymptote to a constant magnetic field, $\mathbf{B}_{0}$, far from the current sheet. A common example is the kinetic Harris equilibrium (Harris 1962; Yamada et al. 2000), with 1-D spatial variation in the direction orthogonal to the current sheet plane (e.g., the plasmasheet in the magnetotail). A uniform out-of-plane guide field, $\mathbf{B}_{g}$, and uniform background (lobe) populations may be added without affecting the self-consistency of the equilibrium.

When the current sheet thickness is on the order of the ion skin depth $\left(c / \omega_{i}\right)$ or less, the Harris equilibrium is unstable to spontaneous reconnection through collisionless tearing instabilities (Drake and Lee 1977) that create $x$-points. The magnetic tension in newly reconnected flux tubes makes them snap away from the $x$-point towards the outflow. The outof-plane induction (reconnection) electric field causes inflow of unreconnected field lines which are topologically converted into reconnected field lines at the $x$-point.

Tearing instabilities are usually very slow (growth rate on the order of 100's of ion inverse cyclotron frequencies, $\Omega_{c i}^{-1}$ ). Kinetic simulations of spontaneous reconnection initiated solely by this instability have been performed (Pritchett 2005), but they can be timeconsuming. To speed things up, simulations of kinetic reconnection are usually driven, either by an initial perturbation which effectively introduces a very small local magnetic field across the thin current sheet or by an $\mathbf{E} \times \mathbf{B}$ drift in the inflow, which thins the current sheet and speeds up the tearing. As an alternative to Harris sheet initialization, force free equilibrium current sheets are sometimes employed (Drake et al. 2003). The initial density can then be uniform because pressure is not necessary to balance magnetic forces. Another strategy for simulating reconnection is to begin with an alreadyreconnected field-line configuration (Pritchett 2007). Still another is to employ kinetic simulations with open boundary conditions allowing boundary inflow of magnetic flux and boundary outflow of reconnected magnetic flux; this can lead to steady state reconnection (Daughton et al. 2006). The rate of steady state reconnection as dictated by geometric properties of the current sheet were analysed early-on (Sweet 1958; Parker 1957; Petschek 1964).

\subsection{Hall Reconnection}

A major advance in modeling collisionless reconnection using MHD and fluid models occurred when it was shown that the Hall term in the generalized Ohm's law was essential to expedite the breaking of field lines (i.e., the breaking of the frozen-in condition in the absence of collisions). This term effectively separates electron physics near the $x$-point from ion physics further away, as sketched in Fig. 6. Work is performed on electrons in the small so-called electron diffusion region, where the electrons are not frozen-in. The electron diffusion region extends from the $x$-point to 10 's of electron inertial lengths, $c / \omega_{e}$, downstream on either side of the $x$-point. Beyond the electron diffusion region the electrons can be frozen-in but the ions are not, forming the socalled ion diffusion region, which is many ion inertial lengths long. Parallel electron currents that form around the separatrices due to flux-tube-widening (Uzdensky and Kulsrud 2006) act as Hall currents which produce a quadrupolar Hall magnetic field, $\mathbf{B}_{H}$, and a Hall electric field, $\mathbf{E}_{H}$, orthogonal to both the $\mathbf{B}_{H}$ and the Hall current. The necessity of including Hall physics has led to the development of Hall MHD (Shay et al. 1999; 
Fig. 6 Current systems and fields found in the Hall reconnection scenario

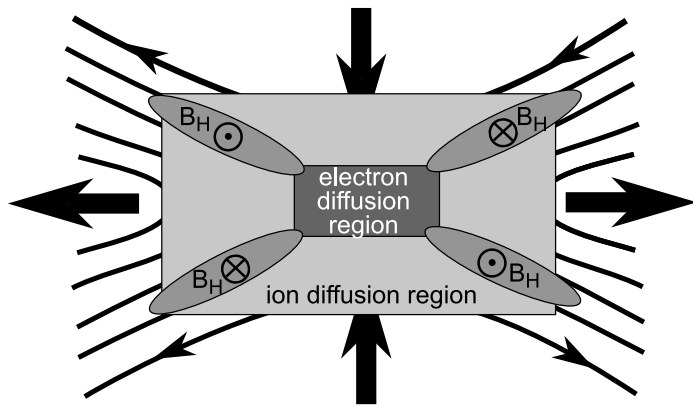

Huba 2005) and to the use of two-fluid and hybrid models (Karimabadi et al. 2004) in reconnection simulations. However it is important to realize that Hall processes are always automatically included in kinetic simulations of reconnection.

\subsection{Kinetic Processes in Reconnection}

PIC simulations and satellite measurements have also revealed a number of intrinsically kinetic processes that occur after reconnection has begun. Electron two-stream instabilities form near the separatrices that separate incoming flux tubes from outflowing reconnected flux tubes. These instabilities saturate by trapping the electron streams, thereby forming electron phase space holes propagating towards and away from the $x$-point. Electron holes have been seen in reconnection simulations (Lapenta et al. 2011) and observed in the tail through their associated bipolar electric fields (Cattell et al. 2002, 2005). Another (different) kind of trapping is associated with electron bounce motion due to magnetic mirror points and bipolar electric fields along flux tubes in the inflow and near-outflow. Analysis of these motions together with temperature anisotropy considerations have led to useful equations of state in the presence of a guide field (Le et al. 2009). There is still another electric fieldthe electrostatic Hall electric field, which is typically as large as $100 \mathrm{mV} / \mathrm{m}$ in the tail. This electric field has been shown to accelerate ions to multi-keV energies in the direction perpendicular to magnetic field lines (Wygant et al. 2005).

Kinetic Alfvén wave physics has been invoked in explaining the fast outflowing Poynting flux corresponding to the Hall electric and magnetic fields (Shay et al. 2011). Electron whistler waves have been identified radiating from the separatrices into the inflowing plasma (Goldman et al. 2012). Efficient electron acceleration at physical ion to electron mass ratios has been studied in kinetic simulations by Ricci et al. (2003), and has been associated with the magnetic stress in contracting curved flux tubes (magnetic islands) (Drake et al. 2006).

\subsection{Guide Field Effects}

Magnetic guide fields, $B_{g}$, can be as large as or larger than $B_{0}$ in Earth's magnetopause. Together with the density gradient across the magnetopause current sheet they can produce diamagnetic drifts that can move $x$-points in the outflow direction and even prevent reconnection (Swisdak et al. 2003). Che et al. (2011) have shown that the thin current sheet of background electrons which forms after the initial Harris current sheet is torn can be unstable to a shear instability in the presence of a moderate $B_{g}$. The instability creates a rightcircularly polarized electromagnetic wave along $\mathbf{B}_{g}$ that saturates by thickening the current sheet, thereby removing its shear free-energy. The unstable waves lead to anomalous viscosity which can speed up reconnection. 
Guide fields of order $B_{0}$ or less can change the flow of electrons along the separatrices, producing a higher density outflow along one axis of the separatrix and a lower density inflow on the other axis, where electron holes become more robust at early times. Even weak magnetic guide fields can have important effects in Earth's magnetotail. A guide field of $B_{g}=0.1 B_{0}$ or less can still create a strong asymmetry in the Hall quadrupole magnetic field (Eastwood et al. 2010). Such a weak guide field can also deflect towards the separatrix the outflowing collimated electron jets found in antiparallel reconnection simulations (i.e., with $\left.B_{g}=0\right)$ (Goldman et al. 2011). Simulations of reconnection with small guide field are quite sensitive to the assumed ion to electron mass ratio. Guide field jet deflections and other effects are much more pronounced when the mass ratio is physical (e.g. 1836) (Goldman et al. 2011).

\subsection{Flux Pile-up and Dipolarization Processes Associated with Collisionless Reconnection}

PIC simulations of magnetotail reconnection initiated with a Harris sheet representing the plasmasheet and lower density background particles representing the lobes can display pileup of reconnected field lines as well as other features of measured dipolarization events (Runov et al. 2009). In the simplest case a single $x$-point arises from an initial perturbation in the simulation. As the tension in the reconnected field lines splits open the Harris sheet, leaving behind low density background plasma, a strong pressure force develops at the boundary of the opening higher density Harris sheet. This pressure force opposes the magnetic stress, thereby causing a pileup of reconnected field lines moving with the outflow as the Harris sheet continues to open. This is (roughly) a moving dipolarization front, although dipolarization physics effects are missing from this simple model (e.g., the shape, extent and motions of the plasmasheet, the connectivity of the magnetic field to Earth's magnetic field, etc.). Dipolarization fronts have been shown to be an important mechanism for energizing the plasmasheet (Hamrin et al. 2012). Simulations have shown that the moving pileup front is a critical boundary that separates the ion-diffusion region from the plasmasheet (Goldman et al. 2012).

\subsection{Multiple Islands, $x$-Points and Flux Tubes in Collisionless Reconnection}

Measurements in the solar wind have provided evidence for very long $x$-lines out of the reconnection plane (Phan et al. 2006). In driven or long-time PIC simulations multiple $x$-points can develop. In two-dimensions these are separated by multiple islands (centered on O-points). Island chains have been studied in simulations (Markidis et al. 2012) and have been found in the solar wind and elsewhere. Multiple islands can be found either on the thin background electron current sheets which form during reconnection or along the separatrix of a primary island. Multiple islands can be unstable to merging instabilities (Pritchett 2007). In pioneering recent massive 3D PIC simulations the islands are found to extend out of the reconnection plane. There they become wiggling flux tubes that can touch each other at different out-of-plane points and even produce secondary reconnections where they touch (Daughton et al. 2011).

\section{Turbulence}

Turbulence is the nonlinear transfer of energy in fluctuations from one scale to another. Typically that transfer proceeds from large scales, where macroscopic motion and interactions 
Fig. 7 Magnetic power spectrum of solar wind turbulence, illustrating the inertial regime in which the power falls off as $f^{-5 / 3}$ and successive steepenings at ion and electron scales (from Alexandrova et al. 2009)

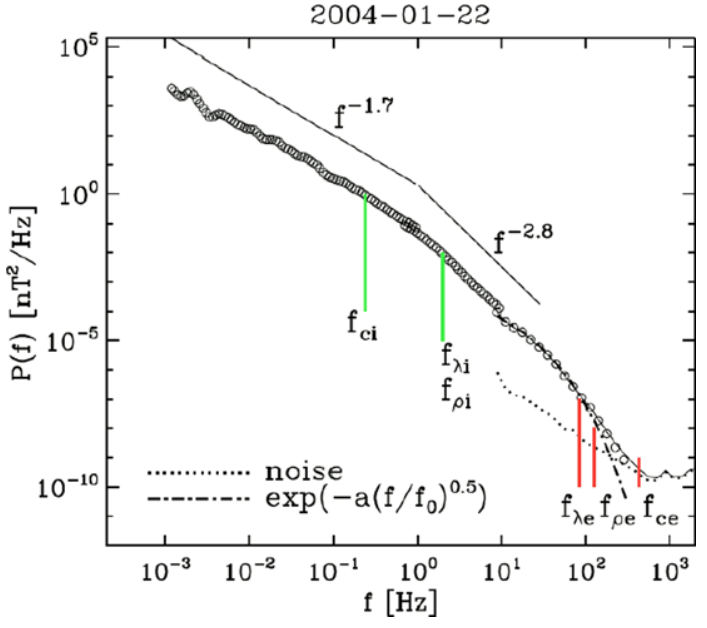

"stir" the medium, toward small scales where eventually the energy is dissipated. Given our earlier discussions about dissipative processes in collisionless plasmas being mediated by microphysics, it should come as no surprise that plasma microphysics is expected to be involved in the turbulent cascade.

However, two aspects complicate plasma turbulence. The first is that there is more than one kinetic scale, and hence one would expect to see signatures of those scales and to examine the contribution each might make to the energy dissipation. Figure 7 shows a typical turbulence spectrum measured in the solar wind (Alexandrova et al. 2009; Sahraoui et al. 2009). Note the Kolmogorov-like $-5 / 3$ spectral slope at lower frequencies. This corresponds to the "inertial range" where energy is cascaded to shorter scales (higher frequencies) without loss in a self-similar fashion. The spectrum steepens beyond frequencies corresponding to typical ion scales and is believed to steepen further or roll-over at electron scales.

The second aspect of the dissipation range of collisionless plasmas is more subtle. Kinetic plasma waves are dispersive, unlike acoustic waves in a fluid. Thus the nonlinear interaction of two waves of similar frequencies will not, in general, lead to a mode at the sum of those frequencies that is a normal mode of the system. The orderly transfer of energy through interactions that are local in frequency (or wave-vector) space which features in the inertial range cannot operate. The nature of the turbulence at these kinetic scales, the local or nonlocal nature of cascaded energy transfer, and the actual dissipation mechanism(s) for that energy are all areas of ongoing research.

One possibility is that the turbulence generates coherent current structures at small scales. These structures, identified through the intermittency properties of the turbulence (Osman et al. 2011), then dissipate through local current-driven instabilities or reconnection. Observations in the solar wind (Phan et al. 2006) and in the turbulent magnetosheath behind the terrestrial bow shock (Retinò et al. 2007; Sundkvist et al. 2007) all provide direct evidence for reconnection events embedded in a turbulent plasma. These events are weak compared to the violent events responsible for solar flares and geomagnetic storms, but may play important roles in plasma heating.

On the larger scale, there must be a fast reconnection mechanism to remove smallscale tangles in the interstellar magnetic field so that the large scale component dominates, as observed (Kulsrud and Zweibel 2008). Possibilities include formation of tiny scale ki- 
netic structures in an overall turbulent background (Servidio et al. 2011), enhancement of the reconnection rate by small scale turbulence in large scale current sheets (Lazarian and Vishniac 1999), breakup of current sheets through instabilities (Loureiro et al. 2007; Huang and Bhattacharjee 2013), and formation of thin current sheets by ion-neutral friction (Brandenburg and Zweibel 1994).

\section{Concluding Remarks}

In this paper we have given an overview of the role microphysical processes play in both solar system and astrophysical plasmas; although we have not discussed laboratory plasmas directly, under collisionless regimes the same remarks would and do apply there. Such processes arise due to the nonequilibrium nature of collisionless media. Although microphysics then controls the transport properties, energy partition (heating, acceleration, etc.), and hence effective "equation of state," the consequences of microphysical control cannot often be captured by retaining the mathematical form of collision-dominated conduction or diffusion. Instead, microphysics acts on discrete portions of velocity space and participates in physics that couples the largest scales to the smallest ones.

The papers that follow in this special edition explore specific applications of microphysics to collisionless plasmas. Many of them also attempt to extrapolate lessons learned in one regime or application to another. Reference to the underlying principles in the present paper will prove helpful in setting those papers in context.

Acknowledgements SJS and EGZ are grateful for the hospitality provided by ISSI during the workshop from which this special issue emerged. EGZ acknowledges support by NSF Grants NSF PHY-0821899 and NSF AST-0909900 to the University of Wisconsin, and the support of CMSO. SJS acknowledges the support of the UK STFC.

Open Access This article is distributed under the terms of the Creative Commons Attribution License which permits any use, distribution, and reproduction in any medium, provided the original author(s) and the source are credited.

\section{References}

O. Alexandrova, J. Saur, C. Lacombe, A. Mangeney, J. Mitchell, S.J. Schwartz, P. Robert, Universality of solar-wind turbulent spectrum from MHD to electron scales. Phys. Rev. Lett. 103(16), 165003 (2009). doi:10.1103/PhysRevLett.103.165003

H. Alfvén, Plasma Universe, in Space Technology Plasma Issues in 2001, ed. by H. Garrett, J. Feynman, S. Gabriel (1986), pp. 373-407

A.R. Bell, The acceleration of cosmic rays in shock fronts. II. Mon. Not. R. Astron. Soc. 182, 443-455 (1978)

A.R. Bell, Turbulent amplification of magnetic field and diffusive shock acceleration of cosmic rays. Mon. Not. R. Astron. Soc. 353, 550-558 (2004). doi:10.1111/j.1365-2966.2004.08097.x

R.D. Blandford, J.P. Ostriker, Particle acceleration by astrophysical shocks. Astrophys. J. Lett. 221, 29-32 (1978). doi:10.1086/182658

P. Blasi, Origin of galactic cosmic rays, arXiv:1211.4799 (2012)

S. Braginskii, Transport processes in a plasma. Rev. Plasma Phys. 1, 205 (1965)

A. Brandenburg, E.G. Zweibel, The formation of sharp structures by ambipolar diffusion. Astrophys. J. Lett. 427, 91-94 (1994). doi:10.1086/187372

D. Burgess, Collisionless shocks, in Introduction to Space Physics, ed. by M.G. Kivelson and C.T. Russell (Cambridge University Press, Cambridge, 1995), pp. 129-163. Chap. 5

C. Cattell, J. Crumley, J. Dombeck, J.R. Wygant, F.S. Mozer, Polar observations of solitary waves at the Earth's magnetopause. Geophys. Res. Lett. 29(5), 050000-1 (2002). doi:10.1029/2001GL014046

C. Cattell, J. Dombeck, J. Wygant, J.F. Drake, M. Swisdak, M.L. Goldstein, W. Keith, A. Fazakerley, M. André, E. Lucek, A. Balogh, Cluster observations of electron holes in association with magnetotail reconnection and comparison to simulations. J. Geophys. Res. 110(A9), 1211 (2005). doi:10.1029/ 2004JA010519 
S. Chapman, V.C.A. Ferraro, A new theory of magnetic storms. Terr. Magn. Atmos. Electr. 36, 171 (1931). doi:10.1029/TE036i003p00171

H. Che, J.F. Drake, M. Swisdak, A current filamentation mechanism for breaking magnetic field lines during reconnection. Nature 474, 184-187 (2011). doi:10.1038/nature10091

G.F. Chew, M.L. Goldberger, F.E. Low, The Boltzmann equation and the one-fluid hydromagnetic equations in the absence of particle collisions. Proc. R. Soc. Lond. Ser. A 236, 112-118 (1956). doi:10.1098/ rspa. 1956.0116

S.W.H. Cowley, Theoretical perspectives of the magnetopause: a tutorial review, in Physics of the Magnetopause, ed. by P. Song, B.U.O. Sonnerup, M.F. Thomsen. Geophysical Monograph Series, vol. 90 (American Geophysical Union, Washington, 1995), p. 29

W. Daughton, J. Scudder, H. Karimabadi, Fully kinetic simulations of undriven magnetic reconnection with open boundary conditions. Phys. Plasmas 13(7), 072101 (2006). doi:10.1063/1.2218817

W. Daughton, V. Roytershteyn, H. Karimabadi, L. Yin, B.J. Albright, B. Bergen, K.J. Bowers, Role of electron physics in the development of turbulent magnetic reconnection in collisionless plasmas. Nat. Phys. 7, 539-542 (2011). doi:10.1038/nphys 1965

R.L. Dewar, Interaction between hydromagnetic waves and a time-dependent, inhomogeneous medium. Phys. Fluids 13, 2710-2720 (1970). doi:10.1063/1.1692854

J.F. Drake, Y.C. Lee, Kinetic theory of tearing instabilities. Phys. Fluids 20, 1341-1353 (1977). doi: $10.1063 / 1.862017$

J.F. Drake, M. Swisdak, C. Cattell, M.A. Shay, B.N. Rogers, A. Zeiler, Formation of electron holes and particle energization during magnetic reconnection. Science 299, 873-877 (2003). doi:10.1126/ science. 1080333

J.F. Drake, M. Swisdak, H. Che, M.A. Shay, Electron acceleration from contracting magnetic islands during reconnection. Nature 443, 553-556 (2006). doi:10.1038/nature05116

J.W. Dungey, Interplanetary magnetic field and the auroral zones. Phys. Rev. Lett. 6, 47-48 (1961). doi:10.1103/PhysRevLett.6.47

J.W. Dungey, Origins of the concept of reconnection and its application to the magnetopause: a historical view, in Physics of the Magnetopause, ed. by P. Song, B.U.O. Sonnerup, M.F. Thomsen. Geophysical Monograph Series, vol. 90 (American Geophysical Union, Washington, 1995), p. 17

J.P. Eastwood, M.A. Shay, T.D. Phan, M. Øieroset, Asymmetry of the ion diffusion region hall electric and magnetic fields during guide field reconnection: observations and comparison with simulations. Phys. Rev. Lett. 104(20), 205001 (2010). doi:10.1103/PhysRevLett.104.205001

T.G. Forbes, E.R. Priest, Photospheric magnetic field evolution and eruptive flares. Astrophys. J. 446, 377 (1995). doi:10.1086/175797

S.P. Gary, Theory of Space Plasma Microinstabilities (Cambridge University Press, Cambridge, 2005)

M.V. Goldman, G. Lapenta, D.L. Newman, S. Markidis, H. Che, Jet deflection by very weak guide fields during magnetic reconnection. Phys. Rev. Lett. 107(13), 135001 (2011). doi:10.1103/PhysRevLett.107. 135001

M.V. Goldman, D.L. Newman, G. Lapenta, S. Markidis, J.T. Gosling, L. Andersson, S. Eriksson, Particle and wave energy transport during magnetic reconnection, AGU Fall Meeting Abstracts, 24-05 (2012)

M. Hamrin, O. Marghitu, P. Norqvist, S. Buchert, M. André, B. Klecker, L.M. Kistler, I. Dandouras, The role of the inner tail to midtail plasma sheet in channeling solar wind power to the ionosphere. J. Geophys. Res. 117(A16), 6310 (2012). doi:10.1029/2012JA017707

E. Harris, On a plasma sheath separating regions of oppositely directed magnetic field. Nuovo Cim. 23(1), 115-121 (1962)

Y.-M. Huang, A. Bhattacharjee, Plasmoid instability in high-Lundquist-number magnetic reconnection, arXiv:1301.0331 (2013)

J.D. Huba, Hall magnetic reconnection: guide field dependence. Phys. Plasmas 12(1), 012322 (2005). doi:10.1063/1.1834592

H. Karimabadi, D. Krauss-Varban, J.D. Huba, H.X. Vu, On magnetic reconnection regimes and associated three-dimensional asymmetries: hybrid, hall-less hybrid, and hall-MHD simulations. J. Geophys. Res. 109(A18), 9205 (2004). doi:10.1029/2004JA010478

A. Kis, M. Scholer, B. Klecker, E. Möbius, E.A. Lucek, H. Rème, J.M. Bosqued, L.M. Kistler, H. Kucharek, Multi-spacecraft observations of diffuse ions upstream of Earth's bow shock. Geophys. Res. Lett. 31, 20801 (2004). doi:10.1029/2004GL020759

M.G. Kivelson, C.T. Russell, Introduction to Space Physics (Cambridge University Press, Cambridge, 1995)

H. Kucharek, E. Möbius, M. Scholer, C. Mouikis, L. Kistler, T. Horbury, A. Balogh, H. Réme, J. Bosqued, On the origin of field-aligned beams at the quasi-perpendicular bow shock: multi-spacecraft observations by cluster. Ann. Geophys. 22, 2301-2308 (2004). doi:10.5194/angeo-22-2301-2004

R. Kulsrud, W.P. Pearce, The effect of wave-particle interactions on the propagation of cosmic rays. Astrophys. J. 156, 445 (1969). doi:10.1086/149981 
R.M. Kulsrud, E.G. Zweibel, On the origin of cosmic magnetic fields. Rep. Prog. Phys. 71(4), 046901 (2008). doi:10.1088/0034-4885/71/4/046901

G. Lapenta, S. Markidis, A. Divin, M.V. Goldman, D.L. Newman, Bipolar electric field signatures of reconnection separatrices for a hydrogen plasma at realistic guide fields. Geophys. Res. Lett. 38, 17104 (2011). doi:10.1029/2011GL048572

A. Lazarian, E.T. Vishniac, Reconnection in a weakly stochastic field. Astrophys. J. 517, 700-718 (1999). doi: $10.1086 / 307233$

A. Le, J. Egedal, W. Daughton, W. Fox, N. Katz, Equations of state for collisionless guide-field reconnection. Phys. Rev. Lett. 102(8), 085001 (2009). doi:10.1103/PhysRevLett.102.085001

M.M. Leroy, A. Mangeney, A theory of energization of solar wind electrons by the Earth's bow shock. Ann. Geophys. 2, 449-456 (1984)

N.F. Loureiro, A.A. Schekochihin, S.C. Cowley, Instability of current sheets and formation of plasmoid chains. Phys. Plasmas 14(10), 100703 (2007). doi:10.1063/1.2783986

S. Markidis, P. Henri, G. Lapenta, A. Divin, M.V. Goldman, D. Newman, S. Eriksson, Collisionless magnetic reconnection in a plasmoid chain. Nonlinear Process. Geophys. 19, 145-153 (2012). doi:10.5194/ npg-19-145-2012

W. Masood, S.J. Schwartz, M. Maksimovic, A.N. Fazakerley, Electron velocity distribution and lion roars in the magnetosheath. Ann. Geophys. 24, 1725-1735 (2006). doi:10.5194/angeo-24-1725-2006

A. Masters, L. Stawarz, M. Fujimoto, S.J. Schwartz, N. Sergis, M.F. Thomsen, A. Retinò, H. Hasegawa, B. Zieger, G.R. Lewis, A.J. Coates, P. Canu, M.K. Dougherty, Electron acceleration to relativistic energies at a strong quasi-parallel shock wave. Nat. Phys. 9, 164-167 (2013). doi:10.1038/nphys2541

L. Matteini, S. Landi, P. Hellinger, F. Pantellini, M. Maksimovic, M. Velli, B.E. Goldstein, E. Marsch, Evolution of the solar wind proton temperature anisotropy from 0.3 to 2.5 AU. Geophys. Res. Lett. 34, 20105 (2007). doi:10.1029/2007GL030920

M.E. McKean, D. Winske, S.P. Gary, Mirror and ion cyclotron anisotropy instabilities in the magnetosheath. J. Geophys. Res. 97, 19421 (1992). doi:10.1029/92JA01842

J.J. Mitchell, S.J. Schwartz, U. Auster, Electron cross talk and asymmetric electron distributions near the earth's bowshock. Ann. Geophys. 30, 503-513 (2012). doi:10.5194/angeo-30-503-2012

Y. Ohira, B. Reville, J.G. Kirk, F. Takahara, Two-dimensional particle-in-cell simulations of the nonresonant, cosmic-ray-driven instability in supernova remnant shocks. Astrophys. J. 698, 445-450 (2009). doi:10.1088/0004-637X/698/1/445

K.T. Osman, W.H. Matthaeus, A. Greco, S. Servidio, Evidence for inhomogeneous heating in the solar wind. Astrophys. J. Lett. 727, 11 (2011). doi:10.1088/2041-8205/727/1/L11

K. Papadopoulos, A review of anomalous resistivity for the ionosphere. Rev. Geophys. Space Phys. 15, 113127 (1977). doi:10.1029/RG015i001p00113

E.N. Parker, Acceleration of cosmic rays in solar flares. Phys. Rev. 107, 830-836 (1957). doi:10.1103/ PhysRev.107.830

H.E. Petschek, Magnetic field annihilation, in Physics of Solar Flares, ed. by W.N. Ness. NASA SP, vol. 50, 1964 , p. 425

T.D. Phan, J.T. Gosling, M.S. Davis, R.M. Skoug, M. Øieroset, R.P. Lin, R.P. Lepping, D.J. McComas, C.W. Smith, H. Reme, A. Balogh, A magnetic reconnection X-line extending more than 390 Earth radii in the solar wind. Nature 439, 175-178 (2006). doi:10.1038/nature04393

P.L. Pritchett, Onset and saturation of guide-field magnetic reconnection. Phys. Plasmas 12(6), 062301 (2005). doi:10.1063/1.1914309

P.L. Pritchett, Kinetic properties of magnetic merging in the coalescence process. Phys. Plasmas 14(5), 052102 (2007). doi:10.1063/1.2727458

A. Retinò, D. Sundkvist, A. Vaivads, F. Mozer, M. André, C.J. Owen, In situ evidence of magnetic reconnection in turbulent plasma. Nat. Phys. 3, 236-238 (2007). doi:10.1038/nphys574

B. Reville, J.G. Kirk, P. Duffy, Steady-state solutions in nonlinear diffusive shock acceleration. Astrophys. J. 694, 951-958 (2009). doi:10.1088/0004-637X/694/2/951

P. Ricci, G. Lapenta, J.U. Brackbill, Electron acceleration and heating in collisionless magnetic reconnection. Phys. Plasmas 10, 3554-3560 (2003). doi:10.1063/1.1598207

M.A. Riquelme, A. Spitkovsky, Magnetic amplification by magnetized cosmic rays in supernova remnant shocks. Astrophys. J. 717, 1054-1066 (2010). doi:10.1088/0004-637X/717/2/1054

A. Runov, V. Angelopoulos, M.I. Sitnov, V.A. Sergeev, J. Bonnell, J.P. McFadden, D. Larson, K.-H. Glassmeier, U. Auster, THEMIS observations of an earthward-propagating dipolarization front. Geophys. Res. Lett. 36, 14106 (2009). doi:10.1029/2009GL038980

F. Sahraoui, M.L. Goldstein, P. Robert, Y.V. Khotyaintsev, Evidence of a cascade and dissipation of solarwind turbulence at the electron gyroscale. Phys. Rev. Lett. 102(23), 231102 (2009). doi:10.1103/ PhysRevLett.102.231102 
S.J. Schwartz, Shocks: commonalities in solar-terrestrial chains. Space Sci. Rev. 124, 333-344 (2006). doi:10.1007/s11214-006-9093-y

S.J. Schwartz, D. Burgess, J.J. Moses, Low-frequency waves in the Earth's magnetosheath: present status. Ann. Geophys. 14, 1134-1150 (1996). doi:10.1007/s00585-996-1134-Z

S.J. Schwartz, T. Horbury, C. Owen, W. Baumjohann, R. Nakamura, P. Canu, A. Roux, F. Sahraoui, P. Louarn, J.-A. Sauvaud, J.-L. Pinçon, A. Vaivads, M.F. Marcucci, A. Anastasiadis, M. Fujimoto, P. Escoubet, M. Taylor, S. Eckersley, E. Allouis, M.-C. Perkinson, Cross-scale: multi-scale coupling in space plasmas. Exp. Astron. 23, 1001-1015 (2009a). doi:10.1007/s10686-008-9085-x

S. Schwartz, S.D. Bale, M. Fujimoto, P. Hellinger, M. Kessel, G. Le, W. Liu, P. Louarn, I. Mann, R. Nakamura, C. Owen, J.-L. Pinçon, L. Sorriso-Valvo, A. Vaivads, R.F. Wimmer-Schweingruber, Cross-scale: multi-scale coupling in space plasma, Assessment Study Report, arXiv:0912.0856 (2009b)

N. Sckopke, G. Paschmann, S.J. Bame, J.T. Gosling, C.T. Russell, Evolution of ion distributions across the nearly perpendicular bow shock-specularly and non-specularly reflected-gyrating ions. J. Geophys. Res. 88, 6121-6136 (1983). doi:10.1029/JA088iA08p06121

J.D. Scudder, A. Mangeney, C. Lacombe, C.C. Harvey, C.S. Wu, The resolved layer of a collisionless, high beta, supercritical, quasi-perpendicular shock wave. III-Vlasov electrodynamics. J. Geophys. Res. 91, 11075-11097 (1986). doi:10.1029/JA091iA10p11075

S. Servidio, A. Greco, W.H. Matthaeus, K.T. Osman, P. Dmitruk, Statistical association of discontinuities and reconnection in magnetohydrodynamic turbulence. J. Geophys. Res. 116(A15), 9102 (2011). doi:10.1029/2011JA016569

M.A. Shay, J.F. Drake, B.N. Rogers, R.E. Denton, The scaling of collisionless, magnetic reconnection for large systems. Geophys. Res. Lett. 26, 2163-2166 (1999). doi:10.1029/1999GL900481

M.A. Shay, J.F. Drake, J.P. Eastwood, T.D. Phan, Super-Alfvénic propagation of substorm reconnection signatures and Poynting flux. Phys. Rev. Lett. 107(6), 065001 (2011). doi:10.1103/PhysRevLett. 107.065001

J. Skilling, Cosmic ray streaming. III-Self-consistent solutions. Mon. Not. R. Astron. Soc. 173, 255-269 (1975)

D. Sundkvist, A. Retinò, A. Vaivads, S.D. Bale, Dissipation in turbulent plasma due to reconnection in thin current sheets. Phys. Rev. Lett. 99(2), 025004 (2007). doi:10.1103/PhysRevLett.99.025004

P.A. Sweet, The neutral point theory of solar flares, in Electromagnetic Phenomena in Cosmical Physics, ed. by B. Lehnert. IAU Symposium, vol. 6, 1958, p. 123

M. Swisdak, B.N. Rogers, J.F. Drake, M.A. Shay, Diamagnetic suppression of component magnetic reconnection at the magnetopause. J. Geophys. Res. 108, 1218 (2003). doi:10.1029/2002JA009726

R.A. Treumann, Fundamentals of collisionless shocks for astrophysical application, 1. Non-relativistic shocks. Astron. Astrophys. Rev. 17, 409-535 (2009). doi:10.1007/s00159-009-0024-2

B.T. Tsurutani, E.J. Smith, R.R. Anderson, K.W. Ogilvie, J.D. Scudder, D.N. Baker, S.J. Bame, Lion roars and nonoscillatory drift mirror waves in the magnetosheath. J. Geophys. Res. 87, 6060-6072 (1982). doi:10.1029/JA087iA08p06060

D.A. Uzdensky, R.M. Kulsrud, Physical origin of the quadrupole out-of-plane magnetic field in hallmagnetohydrodynamic reconnection. Phys. Plasmas 13, 062305 (2006)

V.M. Vasyliunas, Theoretical models of magnetic field line merging. I. Rev. Geophys. Space Phys. 13, 303336 (1975). doi:10.1029/RG013i001p00303

H.J. Voelk, L.O. Drury, J.F. McKenzie, Hydrodynamic estimates of cosmic ray acceleration efficiencies in shock waves. Astron. Astrophys. 130, 19-28 (1984)

D.G. Wentzel, Hydromagnetic waves excited by slowly streaming cosmic rays. Astrophys. J. 152, 987 (1968). doi: $10.1086 / 149611$

C.S. Wu, A fast Fermi process — energetic electrons accelerated by a nearly perpendicular bow shock. J. Geophys. Res. 89, 8857-8862 (1984). doi:10.1029/JA089iA10p08857

C.S. Wu, D. Winske, M. Tanaka, K. Papadopoulos, K. Akimoto, C.C. Goodrich, Y.M. Zhou, S.T. Tsai, P. Rodriguez, C.S. Lin, Microinstabilities associated with a high Mach number, perpendicular bow shock. Space Sci. Rev. 37, 63-109 (1984). doi:10.1007/BF00213958

J.R. Wygant, C.A. Cattell, R. Lysak, Y. Song, J. Dombeck, J. McFadden, F.S. Mozer, C.W. Carlson, G. Parks, E.A. Lucek, A. Balogh, M. Andre, H. Reme, M. Hesse, C. Mouikis, Cluster observations of an intense normal component of the electric field at a thin reconnecting current sheet in the tail and its role in the shock-like acceleration of the ion fluid into the separatrix region. J. Geophys. Res. 110(A9), 9206 (2005). doi:10.1029/2004JA010708

M. Yamada, R. Kulsrud, H. Ji, Magnetic reconnection. Rev. Mod. Phys. 82, 603-664 (2010). doi:10.1103/ RevModPhys.82.603

M. Yamada, F.M. Levinton, N. Pomphrey, R. Budny, J. Manickam, Y. Nagayama, Investigation of magnetic reconnection during a sawtooth crash in a high-temperature tokamak plasma. Phys. Plasmas 1, 32693276 (1994). doi:10.1063/1.870479 
M. Yamada, H. Ji, S. Hsu, T. Carter, R. Kulsrud, F. Trintchouk, Experimental investigation of the neutral sheet profile during magnetic reconnection. Phys. Plasmas 7, 1781-1787 (2000). doi:10.1063/ 1.873999

E.G. Zweibel, Cosmic-ray history and its implications for galactic magnetic fields. Astrophys. J. 587, 625637 (2003). doi:10.1086/368256

E.G. Zweibel, M. Yamada, Magnetic reconnection in astrophysical and laboratory plasmas. Annu. Rev. Astron. Astrophys. 47, 291-332 (2009). doi:10.1146/annurev-astro-082708-101726 